\title{
Repercussões de um programa de atenção psicológica sobre a qualidade de vida de idosas praticantes do método Pilates
}

\section{Repercussions of a psychological care program on the quality of life of elderly practitioners of the method Pilates}
Repercusiones de un programa de atención psicológica en la calidad de vida de los profesionales de mayores del método Pilates

\author{
Emília Cristina Ferreira de Carvalho* \\ Universidade Federal do Vale do São Francisco - UNIVASF, Petrolina, Pernambuco, \\ Brasil
}

\section{Marcelo de Maio Nascimento**}

Universidade Federal do Vale do São Francisco - UNIVASF, Petrolina, Pernambuco, Brasil

\begin{abstract}
RESUMO
O presente estudo tem por fim apresentar a percepção da Qualidade de Vida (QV) de integrantes de um Programa de Atividade Física (PAF), em caráter interdisciplinar, o qual associou o método Pilates com atividades de um Programa de Atenção Psicológica (PAP), fundamentado nas Modalidades de Prática Psicológica (MPP). Trata-se de uma pesquisa descritiva exploratória de abordagem qualitativa, composta por 10 indivíduos do sexo feminino $(64 \pm 2,4$ anos), realizado na cidade de Petrolina-PE. Os dados foram coletados a partir de entrevistas semiestruturadas, submetidas à técnica da Análise de Conteúdo de Bardin, ao final, obteve-se cinco categorias: repercussões físicas e psíquicas, repercussões interpessoais, repercussões espirituais, repercussões de tempo e espaço e repercussões nos hábitos de vida. As narrativas demonstraram que a inclusão das MPP ampliaram os benefícios da prática do exercício físico (EF) à saúde. Conclui-se que os procedimentos interdisciplinares se apresentaram como medida eficiente à promoção da QV. Sendo assim, sugerem-se ações conjuntas entre profissionais de Educação Física e Psicologia na área do envelhecimento ativo do idoso.
\end{abstract}

Palavras-chave: atenção psicológica, envelhecimento, método Pilates, interdisciplinaridade.

\section{ABSTRACT}

This study aims to present the perception of quality of life (QOL) of members of a Program of Physical Activity (PAF) in interdisciplinary character, which joined the Pilates method with activities of a Psychological Care Program (PAP) based on Psychological Practice Implementation (MPP). It is an 
exploratory descriptive study of qualitative approach, consisting of 10 female subjects (64 \pm 2.4 years), in the city of Petrolina-PE. Data were collected from semi-structured interviews were submitted to the technique of Bardin Content Analysis, at the end, five categories were obtained: physical and psychological repercussions, interpersonal consequences, spiritual repercussions repercussions of time and space and impact on habits life. The narratives demonstrated that the inclusion of MPP extended the benefits of physical exercise (PE) to health. It is concluded that the interdisciplinary procedures performed as efficient measure to promote the QV. Thus, joint actions are suggested between physical education and psychology professionals in the area of the active ageing of the elderly.

Keywords: psychological attention, aging, pilates method, interdisciplinarity.

\begin{abstract}
RESUMEN
Este estudio tiene como objetivo presentar la percepción de calidad de vida (QV) de los miembros de un Programa de Actividad Física (PAF) de carácter interdisciplinario, que se unió el método Pilates con actividades de un Programa de Atención Psicológica (PAP) basado en Aplicación Práctica Psicológica (APP). Se trata de un estudio descriptivo exploratorio de abordaje cualitativo, que consta de 10 sujetos femeninos (64 $\pm 2,4$ años), en la ciudad de Petrolina. Los datos se obtuvieron de entrevistas semiestructuradas fueron sometidos a la técnica de análisis de contenido de Bardin, al final, se obtuvieron cinco categorías: repercusiones físicas y psicológicas, consecuencias interpersonales, repercusiones espirituales repercusiones del tiempo y el espacio y el impacto en los hábitos vida. Los relatos demostraron que la inclusión de APP extendió los beneficios del ejercicio físico (EF) para la salud. Se concluye que los procedimientos interdisciplinares realizados como medida eficaz para promover el QV. Así, se sugieren acciones conjuntas entre educación física y profesionales de la psicología en el área de envejecimiento activo de la tercera edad.
\end{abstract}

Palabras clave: atención psicológica, envejecimiento, método Pilates, interdisciplinariedad.

\title{
1 I ntrodução
}

Nas últimas décadas, o envelhecimento populacional vem apresentando características sui generes. $O$ fato advém de transformações na estrutura etária, ou seja, aumento da expectativa média de vida (Santos, Souza, Marinho, \& Mazo, 2013), aliado aos avanços da ciência na área da saúde e queda dos índices de fecundidade e mortalidade (ONU, 2005). Lima e Bittar (2012) apontaram que no ano de 2025, o Brasil apresentará a sexta maior população idosa do mundo. Sendo assim, teremos cerca de 32 milhões de habitantes em idade acima de 60 anos, caracterizando "um país novo de cabelos brancos".

Diante disso, cresce o interesse de distintas áreas do conhecimento para desvendar as variáveis intrínsecas ao processo do envelhecimento humano, o qual não é visto como um estado de vida, mas sim como um processo particular, inevitável e progressivo do 
homem. Até hoje, ainda não foi possível datar, com exatidão, o início do processo do envelhecimento (Cancela, 2007). Porém, o que se sabe é que envelhecer significa conviver, diariamente, com transformações de caráter biopsicossocial, funcionais (Rowe \& Kahn, 1997), além do surgimento de problemas intergeracionais (Braga Cuba, Carvalho, Evangelista, \& Camara, 2015).

De acordo com Araújo e Ceolim (2007), o envelhecimento atinge, progressivamente, a capacidade de adaptação do homem ao meio ambiente. Isso significa dizer que tais transformações serão responsáveis por dificuldades à realização de atividades de vida diária (AVD). Por outro lado, sabe-se também que algumas pessoas, em idade avançada, diferentemente de outras, possuem autonomia para deliberar seus interesses e guiar suas próprias vidas por um longo tempo. Nessa perspectiva, Veras (2009) salienta que a idade cronológica não deve ser considerada como uma barreira à vida; sendo assim, o importante é ampliar os níveis de saúde, acrescentando qualidade aos anos vividos.

Junto ao processo do envelhecimento humano surge o termo qualidade de vida (QV), o qual vem crescendo em atenção e interesse no âmbito político, especialmente em sentido à promoção da saúde de cidadãos idosos. Nessa perspectiva, compreende-se que possuir bons níveis de QV seja algo fundamental à prevenção dos fatores responsáveis pelo processo saúde-doença (Seidl, 2004). Entre estudiosos, o termo QV não apresenta um consenso (Paschoal, 2000), visto que decorre de um conjunto de fatores de ordem biológica, funcional, psicológica, sociocultural, estando, igualmente, atrelado às condições ambientais em que a pessoa vive (Nery, 1993). Assim, pode-se dizer que a QV é multifatorial, implicando na percepção situacional de vida da pessoa.

Diante disso, surge o compromisso de políticas públicas de saúde internacionais, nacionais e regionais em buscar alternativas que viabilizem o envelhecimento humano com qualidade (Serbim \& Figueiredo, 2011). Uma alternativa, de baixo custo e bem aceita pela população idosa para ampliar os níveis da QV incide na prática regular e orientada do exercício físico (EF). Seus benefícios à manutenção e/ou desenvolvimento de capacidades físicas, cognitivas e emocionais, em idade avançada, são conhecidos (Rowe \& Kahn, 1997; Matsudo, 2006). A medida é importante, visto que o envelhecimento trás consigo a perda progressiva de aptidões físicas e funcionais, alterações emocionais, além de agravar uma série de doenças crônico-degenerativas (Alves, Mota, Da Cunha Costa, \& Bezerra Alves, 2004). De acordo com Saba (2001), ao se exercitar o indivíduo ganha em bem-estar físico e mental, ampliando assim seus níveis de QV.

$\mathrm{Na}$ atualidade, dentre as atividades físicas mais recomendadas à população idosa há o método Pilates. Criado por Joseph Hubertus 
Pilates (1880-1967), o método incide em um conjunto de exercícios que buscam o equilíbrio entre a relação corpo-mente. Os exercícios priorizam o aprendizado da consciência da movimentação corporal, em sentido à postura correta, sendo cada vez mais, influenciado pelo pensamento holístico. Diante disso, sua prática é capaz de ampliar os níveis de aptidão física/funcional (Pilates, 1998; Coriolano Appell, Perez, Nascimento, \& Appell Coriolano, 2012); logo de saúde dos praticantes.

Em se tratando da promoção da atividade física (AF) e o idoso há uma interessante questão junto ao caso: em geral grupos de idosos praticantes de exercícios físicos são compostos essencialmente por mulheres. Pereira, \& Okuma (2009) salientaram que, em idade avançada, a feminização é uma particularidade da população brasileira. De acordo com Chaim, Raimundo, Sant'anna, \& Yuaso (2010), na comparação entre os sexos, nos anos finais de vida, mulheres perdem, com maior frequência, o companheiro, sofrendo assim com o isolamento social. Assim, sua participação em programas de atividade física (PAF) é mais comum. Diante disso, a aderência de idosas nos PAFs não se limita à promoção da saúde, visto que aliado ao esporte há a busca de tempo e de espaço à escuta e ao estabelecimento de novos vínculos de amizade.

Considerando a relevância do tratamento da saúde da pessoa idosa, a partir de metodologias abrangentes e integrativas, bem como a necessidade da disseminação de conhecimentos que adotem medidas interdisciplinares de baixo e alto impacto, o presente estudo apresenta as repercussões de um Programa de Atenção Psicológica (PAP), fundamentado nas Modalidades de Prática Psicológica (MPP), sobre a qualidade de vida (QV) de um grupo de idosas praticantes do exercício físico (EF), método Pilates. A ação foi intitulada como "Saúde e Qualidade de Vida do Idoso", uma linha de extensão do "Projeto Integrar" (Edital PROEXT/2013), criado pelo curso de Psicologia da Universidade Federal do Vale do são Francisco, em Petrolina-PE, com as alunas do grupo "Pilates e o Idoso", curso de Educação Física.

\section{Qualidade de vida e interdisciplinaridade}

Conforme "THE WHOQOL GROUP" (1995), a QV incide na percepção do indivíduo sobre sua condição de vida. Inserido neste contexto estão os relacionamentos sociais, objetivos e expectativas de vida, além do estado biopsicossocial da pessoa. Assim trabalhar a QV significa desvincular 0 indivíduo de um modelo absoluto, considerando o conjunto de analogias intrínsecas ao processo. Nessa perspectiva, também se faz interessante ponderar as histórias de vida da pessoa, as quais se apresentam tanto evidentes, como 
ocultas. Porém, para tanto, os profissionais da área da saúde devem dispor de procedimentos que permitam o acesso a essas informações, preferencialmente, por meio de dinâmicas que motivem a pessoa a se expressar de forma voluntária (Barreto, 2009).

Para Seidl (2004), a QV é legitimada a partir de quatro dimensões/fatores. A primeira incide na percepção do indivíduo sobre sua condição física. A segunda refere-se à forma como a pessoa percebe sua condição afetiva e cognitiva. A terceira diz respeito à avaliação de seus relacionamentos e papéis sociais assumidos ou perdidos. Já a quarta versa sobre aspectos intrínsecos ao ambiente em que ela vive. Considerando isso, é possível concluir que a QV é um tema abrangente. Sendo assim, o trabalho nesta área necessita de conhecimentos amplos, eminentemente interdisciplinares. A questão justifica a inclusão de profissionais e procedimentos metodológicos oriundos de diferentes áreas do conhecimento humano em ações do envelhecimento ativo, ou seja, mediante a prática da atividade física (AF). Nessa perspectiva, Gattás \& Furegato (2006, p. 325) salientam:

(...) interdisciplinaridade pode ser entendida como qualquer forma de combinação entre duas ou mais disciplinas, objetivando-se a compreensão de um fim a partir da confluência de pontos de vista diferentes, cujo objetivo final seria a elaboração de síntese relativa ao caso; implica alguma reorganização do processo ensino/aprendizagem e supõe trabalho contínuo de cooperação entre os professores envolvidos.

Atualmente, ações desenvolvidas na área da Educação Física à promoção da QV com a população idosa têm priorizado o trabalho interdisciplinar (Salin, Mazo, Cardoso, \& Garcia, 2011). A medida se fundamenta na busca de um serviço diferenciado para o tratamento da multifatorialidade do processo do envelhecimento humano. Desse modo, uma parceria que vem surtindo bons resultados, incide no trabalho conjunto entre profissionais das áreas da Educação Física e Psicologia (Nascimento, Carvalho, Souza, \& Andrade, 2015), em Programas de Atividade Física (PAF) (Benedetti, Gonçalves, \& Mota, 2007). Os PAFs têm por fim transformar hábitos de vida diários, principalmente, aqueles que possam contribuir negativamente à saúde e bem-estar da pessoa. Sendo assim, medidas interdisciplinares são vistas como ferramentas úteis para o retardo e/ou impedimento da incidência de doenças crônicas degenerativas e mentais (Benedetti, Mazo, \& Borges, 2012); além de trazerem inovações para os processos.

Desde 2013, na Universidade Federal do Vale do São Francisco/Univasf, a prática do $\mathrm{EF}$, método Pilates (60 minutos), é 
oferecido à comunidade idosa associado com atividades de Atenção Psicológica (60 minutos), tendo como base as Modalidades de Prática Psicológica. As atividades interdisciplinares estão sob a responsabilidade de alunos dos cursos de Educação Física e Psicologia, sob a coordenação de professores dos respectivos cursos. A metodologia adotada foi intitulada como PAF \& PAP (Programa Atividade Física \& Programa de Atenção Psicológica) (Nascimento et al., 2015). As atividades são fundamentadas a partir da escuta, do acolhimento e do incentivo à expressão e interação social do cidadão idoso.

\section{Método Pilates e modalidades de prática psicológica}

De acordo com Spirduso (2005), Benedetti, Mazo, \& Borges (2012), mediante a prática do exercício físico a pessoa idosa pode ampliar seus níveis de força muscular, o fluxo sanguíneo, reduzindo o percentual de gordura corporal e de glicose, baixando os níveis da pressão arterial, além de melhorar a densidade corporal óssea. Matsudo (2006) salienta sobre as contribuições da prática do EF à saúde mental do idoso. Segundo a autora, a participação em grupos de atividade física (AF) se consubstancia em medida de valor à busca do equilíbrio corpo-mente. Saba (2001) menciona os benefícios da prática do EF à promoção da qualidade de vida e bem-estar. O fato estaria correlacionado à liberação de hormônios, responsáveis pela sensação de leveza, conforto físico e mental, bem como do dever cumprido. Isso tudo coopera para a sensação de prazer e satisfação pessoal; algo fundamental no processo de autoestima e autoimagem do idoso.

Nessa perspectiva de saúde e longevidade da pessoa idosa por meio da prática do EF surge o método Pilates. O Pilates é definido como arte de controle consciente da movimentação corporal, ou seja: "contrologia" (Comunello, 2011). Seus exercícios são fundamentados em considerações anatômicas, fisiológicas e cinesiológicas. A elaboração e execução dos exercícios tem base em seis princípios: concentração, controle, centralização, fluidez, respiração e precisão (Latey, 2001). Mediante a prática do Pilates, há o treinamento e domínio do "centro de força" corporal, chamado por "powerhouse" (Aparício \& Pérez, 2005).

Conforme o próprio Pilates, esta expressão se refere a - casa de força - circunferência da cintura pélvica que funciona como estrutura de suporte e reforça para a coluna vertebral (Muscolino \& Cipriani, 2004). Os principais benefícios para o organismo humano incidem no aprimoramento da extensibilidade de músculos e tendões, aumento da flexibilidade a nível articular, benfeitorias para o equilíbrio corporal estático e dinâmico, prevenindo quedas, lesões e evitando o óbito 
dessa população (Coriolano Appell et al., 2012). Sendo assim tais ganhos proporcionados pela prática do Pilates são determinantes à promoção da QV nessa faixa etária.

Outra forma de promoção da QV da pessoa idosa incide em sua participação em dinâmicas fundamentadas pelas Modalidades de Prática Psicológica (MPP). De acordo com Barreto (2009), nos últimos anos, a própria prática terapêutica tem ampliado o uso das MPP, visto que elas permitem a articulação entre a clínica e o social. Com as MPP, o fazer psicológico adquire novas perspectivas, uma vez que suas técnicas possibilitam a consideração do indivíduo conexo ao contexto social em que ele se encontra. Entretanto, segundo o autor, as MPP ainda carecem de maior aprofundamento. Isso significa dizer que estudos investigativos, bem como relatos de casos sobre procedimentos metodológicos com as MPP trazem avanços para o mundo acadêmico.

Neri (2004) considerou a abordagem psicológica como uma medida importante para o tratamento de questões do envelhecimento humano, visto que oferece mecanismos distintos dos convencionais, trazendo alternativas inovadoras à intervenção clínica e social. Nessa perspectiva, com as MPP o Psicólogo dispõe de ótimos mecanismos para auxiliar indivíduos a perceberem e aprenderem acerca de pontos/situações conflitantes em suas vidas. No caso da pessoa idosa, o trabalho executado por intermédio de oficinas criativas e de grupos de conversação pode ser visto como tempo e espaço para que os envolvidos avaliem e/ou regulem circunstâncias de vida.

O trabalho com o idoso requer artifícios próprios. Segundo Neri (2004), no âmbito da formação do Psicólogo, por exemplo, é importante que os responsáveis por cursos de formação e programas de especialização contribuam para o destroncamento das práticas tradicionais. A utilização dessa estratégia estimula o desenvolvimento de novos modos de atenção à pessoa, os quais poderão repercutir no avanço da prática do próprio Psicólogo. Chaimowicz (1997) destaca a importância da discussão sobre os modos de atuação do Psicólogo junto ao público longevo, em cursos de graduação e especialização da área da saúde. Para o autor é necessário que as formações considerem e incorporem artifícios para que os profissionais possam melhor planejar, estruturar, executar e avaliar as MPP. Exemplos dessa ordem e, comuns na área da Psicologia, são os grupos operativos e terapêuticos, bem como as oficinas de criatividade. Todos consistem em ações de baixo custo e alta eficiência, direcionados ao acolhimento das necessidades individuais sob a forma grupal.

Em uma caracterização sobre as MPP, Bastos (2010) apresentou os grupos operativos como instrumento propício à promoção da aprendizagem sob a forma de processo. Segundo o autor, a aprendizagem suscitada pelas dinâmicas grupais, algo típico das MPP, 
possibilita que o conhecimento pessoal frente a si e o outro seja (re)elaborado. No caso do grupo terapêutico, cada indivíduo analisa seu próprio sentimento e comportamento, aprendendo a escutar e ser escutado em contextos de interação afetiva. Essa atitude oportuniza a reflexão de aspectos inerentes a si mesmo. Muitas vezes, os depoimentos que surgem trazem subsídios à compreensão de episódios responsáveis pela liberação de sentimentos diversos, todos determinantes a QV (Benevides, Pinto, \& Cavalcante, 2010). Já as oficinas de criatividade têm como interesse a construção de um espaço para o estabelecimento de encontros transformadores (Cupertino, 2006), baseados no olhar e na escuta. A técnica busca a elaboração da experiência pessoal e coletiva a partir do uso de recursos expressivos, fundamentados na linguagem do corpo, do canto, da leitura, das artes, ou seja, de uma sensibilidade, até então, despercebida pela pessoa.

Segundo Cupertino (2006), as MPP que trabalham sob a forma de grupos se destacam por desenvolverem o acolhimento da experiência do outro, permitindo a reunião dos envolvidos a partir de tarefas comuns. A base do trabalho se processa no respeito às limitações de cada um, seguido pela valorização das potencialidades subjetivas.

\section{Método}

Trata-se de um estudo descritivo exploratório de abordagem qualitativa. Segundo Flick (2009a), esta metodologia reproduz os objetos no próprio contexto em que transcorrem, sendo a entrevista seu principal instrumento. Por meio dos questionamentos os entrevistados são estimulados a expressar as percepções sobre si, bem como sobre o mundo que os circunda (Lamnek, 1989). Ao longo das narrativas, o investigador é confrontado com a realidade interna do grupo/pessoa, o que lhe permite abranger, de forma aprofundada, a subjetividade e o contexto social dos entrevistados.

\subsection{Participantes}

A amostra foi composta por 10 indivíduos do sexo feminino $(64 \pm 2,4$ anos), integrantes do PAF, intitulado "Pilates e o Idoso". Os idosos praticaram duas vezes por semana, durante 60 minutos, exercícios do método Pilates, antecedidos por 60 minutos de atividades fundamentadas nas MPP. Os conteúdos do Pilates foram estabelecidos a partir de 15 exercícios do método, todos de fácil execução, destinados ao fortalecimento e estabilização de músculos da região do core. Uma vez por semana foi acrescentada a bola suíça às sequências de exercícios. As dinâmicas das MPP foram desenvolvidas por meio de técnicas de recorte de jornais, revistas, colagem, 
pintura, bordado, conversações e debates; além do canto, acompanhado por violão.

A ação foi desenvolvida nas dependências do Colegiado de Educação Física da Universidade Federal do Vale do São Francisco, na cidade de Petrolina-PE. As atividades estiveram sob a responsabilidade de uma acadêmica de Educação Física ( $6^{\circ}$ semestre) e outra do curso de Psicologia $\left(8^{\circ}\right.$ semestre), coordenadas por professores dos respectivos cursos. Considerou-se como critério de inclusão estar matriculado no PAF, ter uma frequência, mensal mínima, nas atividades de $75 \%$, bem como aceitar participar voluntariamente do estudo e ter assinando o Termo de Consentimento Livre e Esclarecido (TCLE). Foram excluídos indivíduos que apresentaram frequência inferior a $75 \%$ nas atividades ou não assinaram o TCLE.

\subsection{Procedimentos de coleta e análise dos dados}

As entrevistas seguiram o formato semiestruturado (Flick, 2009a; Stake, 2011), regidas por questões norteadas pelo referencial teórico deste estudo, respeitando a metodologia de escuta e escrita sistemática, gravadas em notebook da marca Compaq. Já a transcrição do material seguiu 0 processo de tematização (categorização), indicado por Flick (2009b). Segundo Gibbs (2009), ao tematizar concebemos o caráter fenomenológico às narrativas, captando assim algo a mais da percepção do mundo vivido pelo sujeito. Ao final, os dados foram julgados por meio da "Análise de Conteúdo" de Bardin (2004), que é caracterizada por ligações estabelecidas entre as situações que o sujeito expôs $e$ as manifestações semântico-sintáticas da superfície discursiva.

\subsection{Procedimentos éticos}

O estudo foi aprovado pelo Comitê de Ética da Universidade.

\section{Resultados e discussão}

Os depoimentos obtidos serão apresentados e discutidos a partir de cinco categorias de análise, são elas: i) Repercussões Físicas e Psíquicas: neste grupo semântico encontramos discursos relativos às contribuições da prática do Pilates para o corpo e sua capacidade funcional; além de benefícios das dinâmicas psicossociais para o corpo, a mente e o comportamento das idosas; ii) Repercussões Interpessoais: esta categoria simplifica o conjunto de reverberações proporcionadas pelas atividades interdisciplinares, em sentido integrador ao contexto social dos envolvidos; iii) Repercussões Espirituais: contextualiza aspectos místicos liberados por meio da 
participação das idosas nas atividades do programa de extensão universitária; iv) Repercussões de Tempo e Espaço: caracteriza a identificação temporal e espacial da pessoa com/no programa; e v) Repercussões nos Hábitos de Vida: caracterizando processos reflexivos suscitados pelas atividades do programa, os quais contribuíram para o desenvolvimento de uma visão crítica das idosas frente ao estado pessoal de saúde, seguido do (re)conhecimento da necessidade de transformações do estilo de vida.

\subsection{Repercussões físicas e psíquicas}

Mediante a análise das narrativas se observou indícios de como a prática do Pilates associado às MPP auxiliou as idosas a perceberem o estado de sua capacidade funcional e emocional. A idosa M. E. P. N. relata as vantagens advindas do trabalho conjunto entre a acadêmica Psicóloga e a acadêmica de Educação Física. Segundo ela, a sintonia profissional foi essencial para o acordo de seu corpo com a mente:

“Iniciar as atividades pela atenção psicológica faz com que eu tenha um melhor desempenho nas aulas de atividade física, porque muitas vezes a gente chega aqui com problemas pessoais, trancada, com travas no corpo, aí a gente se fecha para qualquer possibilidade de melhora e cuidado" (M. E. P. N.).

Conforme Rauchbach (2001), ao longo dos anos, a harmonização da pessoa com seu corpo e mente tende a enfraquecer. $O$ caso sobrevém tanto de alteração biopsicofuncionais (Rowe \& Kahn, 1997), como da incorporação de novas atitudes, hábitos e valores de vida. De tal modo, em se tratando da ampliação dos níveis da QV, quanto mais anos vividos, maior a necessidade de ampliar os procedimentos metodológicos à consideração das circunstâncias de vida da pessoa.

Seguindo, as narrações a idosa M. C. S. M. oferece faz um interessante relato sobre a saúde integral. Suas palavras confirmam que a prevenção em saúde pode ser vista, entre outros, como o desenvolvimento de mecanismos à criação e adequação de condições para que forças da própria pessoa pactuem na busca do seu estado de equilíbrio e harmonia:

"Eu acho que as atividades daqui são muito boas, porque além do Pilates, tem atividades que são diferentes, que ajudam na memória, nas emoções, no divertimento. $E$, você sabe né... tudo que trabalha com a gente por inteiro, tem grandes melhoras para nossa vida". (M. C. S. M.). 
De acordo com Pelizzoli (2013), a visão integrativa de saúde surge quando a pessoa reconhece a existência e o valor de forças curativas inerentes aos organismos vivos, muitas intrínsecas a ela própria. Assim, o processo inicia-se quando o indivíduo reconhece que ativar essas forças pode ser útil para o aprimoramento de sua QV e bemestar. Nisso, evidencia-se a indissociabilidade entre corpo-mente, algo valorizado nos procedimentos interdisciplinares da ação de extensão "Pilates e o Idoso".

\subsection{Repercussões interpessoais}

Rizzolli \& Surdi (2010) colocam que o crescimento da população idosa vem oportunizando, cada vez mais, a criação de espaços à convivência, à promoção e à manutenção da "velhice ativa". Algo fundamental à crença de que é possível viver bem com o avanço da idade. O processo de uma vida ativa, em idade avançada, encontrase atrelado, entre outros, na convivência, ou seja, na troca periódica de informações entre pessoas (Matsudo, 2006; Benedetti et al., 2007; Benedetti et al., 2012). Um exemplo disso, foi observado na fala de M. C. S. M: "Hoje eu já brinco mais com os meus netos, saio mais de casa, converso mais com minha família e minhas colegas do Pilates e da igreja".

As ações PAF \& PAP também propiciaram a troca e convivência social além dos muros da universidade, a partir dos relacionamentos intergeracionais: "Eu sempre saio daqui sabendo mais uma coisa nova e ai conto isso pra minha família, netos e para os meus vizinhos" (T. M. S). De acordo com Borges e Magalhães (2011), o relacionamento do idoso com pessoas de faixas etárias diferentes da sua, afigura-se como estratégia à manutenção e ampliação do seu equilíbrio psicossocial.

Observou-se também neste estudo, indivíduos que chegaram as atividades PAF \& PAP com baixos índices de autoestima e autoimagem. Portanto, é importante ressaltar que afetações e sentimentos vivenciados pelo idoso, ao longo de sua vida, podem ser os responsáveis por atitudes introspectivas, o que, consequentemente, vem privá-lo de buscar o contato social. Assim, o idoso deixa de ser requisitado, principalmente, pelos mais jovens. Em contrapartida, há aqueles que assumem comportamentos ativos, que buscam revitalizar antigas amizades, que consolidam novos laços, amadurecendo experiências do ontem no hoje, perpetuando, em concordância com as limitações da idade, o que ainda é possível (Yokoyama, Carvalho, \& Vizzoto, 2006):

"Antes eu me sentia velha, doída do corpo e triste na cabeça, hoje, depois de vir prá cá eu sei que sou outra pessoa! É bom... Quando a idade chega, a gente já não entende mais essas 
coisas de computador e tudo tão rápido e ligeiro. Os jovens não têm tempo para explicar essas coisas; aí agente se entoca (risos). Mas aqui há muita gente jovem que explica as coisas pra gente e isso é ótimo. Dá uma felicidade imensa, sabe?". (M. E. P. N).

Em um contexto de interação afetiva criada pelo próprio grupo, as dinâmicas de MPP ofertaram ao grupo ferramentas para análise dos sentimentos e comportamentos. Nessa perspectiva M. E. P. N descreve: "As atividades psicológicas me ajudam a compartilhar dificuldades, fazer novas amizades, confiar em quem está próximo a mim (...) até com minha família eu mudei".

Sabe-se que dentre as perdas típicas do processo do envelhecimento se encontram a dos laços sociais, determinadas, muitas vezes, pela aposentadoria. Incluso a isso, há a morte de amigos e/ou de companheiros (Leloup \& Hennezel, 2003). Tais mudanças na vida podem gerar distúrbios psíquicos, em certos casos, somatizados. 0 depoimento de M. F. A salienta aspectos de solidão, sanados a partir de seu ingresso no programa de extensão:

“Em casa eu não tenho com quem tá conversando sobre meus problemas, e aqui eu tenho. Além disso, as atividades são divertidas e me deixam sempre mais alegre! Aqui eu conheci muitas pessoas, já tenho muitas colegas com quem converso e que me ajudam a me sentir bem. Antes não era assim, eu estava muito só" (M. F. A.).

Reflexões como as de M. F. A. trazem subsídios à compreensão do início de um estado depressivo; algo determinante à baixa da percepção da QV e do bem-estar da pessoa idosa.

\subsection{Repercussões espirituais}

Batista (2010) aponta que independente de qualquer experiência religiosa, a espiritualidade faz parte da constituição de todos os homens, apresentando-se como ferramenta à prática da atenção à saúde básica. Segundo ele, ela permite o fortalecimento das relações humanas sob a forma dialogada. A técnica se consubstancia na escuta do sensível da pessoa, no incentivo à solidariedade e na valorização de sua dignidade, tudo de forma simples e natural, alcançado, muitas vezes, pela oferta de um abraço ou palavra afetiva. Com isso, já é possível dar início à construção de um caminho rumo ao estabelecimento do equilíbrio físico e mental (Vasconcelos, 2004). 
No discurso de M. E. P. N. se encontra evidencias de como a participação no projeto contribuiu para o fortalecimento de sua espiritualidade:

“Deus levou o meu marido e então o sentido da vida pra mim se perdeu, até mesmo da minha fé. Mas agora, com este espaço me sinto mais próxima de Deus e se estou próxima dele, estou perto do meu marido também" (M. E. P. N).

Batista (2010) afirma que a espiritualidade é uma força capaz de auxiliar o indivíduo, sua família e até mesmo uma comunidade à superação das dificuldades impostas pela vida. Em casos de doença, por exemplo, a espiritualidade trás substrato para o enfrentamento. Elementos dessa ordem foram referidos no discurso de M. I. S. B., quando coloca:

"A fé, muitas vezes, me ajuda a ter força para viver bem, mas agora participando de todas essas atividades, vejo que tudo é um complemento para minha saúde. Eu tenho para mim que estar aqui, às vezes me ajuda a entrar no meu eu mais profundo".

Por intermédio do trabalho em grupo, algo característico da prática esportiva, firmado pela prática do Pilates e consolidado nas dinâmicas das MPP se propiciou o fundamento para o enfrentamento de realidades, que sempre são desafiadoras. Dentre elas, situações de solidão e de tristeza, ligadas às fatalidades da vida, sobrevindas, muitas vezes, da perda de entes queridos, da saída dos filhos de casa e do próprio processo biológico do envelhecimento.

\subsection{Repercussões de tempo e espaço}

Araújo, Coutinho e Carvalho (2005) afirmam que as práticas sociais nos grupos de convivência de idosos cooperam para que seus integrantes sejam incentivados a reafirmar e/ou aprender seu papel de cidadãos. Paralelo a isso, os grupos também se afiançam como espaço aberto ao acolhimento, à escuta e ao exercício do direito à felicidade (Luz \& Amatuzzi, 2008). Sendo assim, M. E. P. N. narra: "Aqui nesse projeto existe um espaço para conversar, dialogar e falar daquilo que nos incomoda; é como uma forma de estar desabafando, sabe?".

As atividades PAF \& PAP também incidiram em tempo e espaço cedido pelos membros da equipe aos idosos; algo que foi reconhecido pelas idosas: "Todos que trabalham aqui tem muito cuidado com a gente, no sentido de considerar nossas limitações e dificuldades e assim vão, aos poucos, nos ajudando a ter mais saúde". Referências 
quanto ao ambiente criado pelo projeto seguem: “(...) aqui tá vendo, é um lugar público e somos tão bem cuidadas, isso vale muito pra gente, a atenção é algo que não têm preço. Aqui a gente tem tempo pra gente" (J. S. M.) .

\subsection{Repercussões nos hábitos de vida}

Entre os principais objetivos de um PAF há a transformação de hábitos de vida, ou seja, a adoção de medidas salutares nas rotinas diárias. Vestígios dessa ordem são encontrados na narrativa de J. S. M.: “( ...) também aprendi a cuidar de mim mesmo, porque agente cuida tanto da família e da casa que se esquece da gente, e que também precisamos de ajuda e cuidados". Pode-se dizer que as dinâmicas realizadas nas MPP complementaram, consubstancialmente, os objetivos da prática do $E F$, em sentido à transformação de hábitos de vida: “Eu vejo que hoje tenho a vontade de ter uma qualidade de saúde melhor, vamos dizer assim, envelhecer com qualidade de vida melhor" (J. S. M).

Ao longo de oito meses de atividades, as dinâmicas das MPP buscaram motivar as idosas do PAF à reflexão e, caso necessário, à transformação de hábitos de vida. Na fala de T. M. S. se observa a visão desenvolvida pelo grupo sobre os procedimentos metodológicos empregados: "Aqui o melhor é que não se dá conselho, se orienta de verdade. As orientações são boas e nos ajudam a melhorar de vida". Rizzolli \& Surdi (2010) afirmam que nos grupos que trabalham com o idoso é relevante à implantação de uma cultura que valorize a análise dos serviços. O caso merece atenção, pois isso é fundamental para que os idosos se sintam acolhidos e retornem.

O tempo ocioso consiste em fator preponderante à queda dos níveis da QV da pessoa idosa (Vieira \& Boeckel, 2012). De acordo com Yokoyama et al. (2006), para o idoso, ocupar-se com algo se apresenta como um modo distinto para alcançar o bem-estar psicológico: “( ...) aqui a gente é colocado numa situação de lutar pela vida, de buscar ter forças e enfrentar as dificuldades como sendo degraus de escadas que a gente sobe para chegar numa grandeza que é a vida com saúde e prazer" (T. M. S.). Dessa forma, entendese que a associação entre a prática do EF com as MPP fortaleceu o desejo do grupo para assumir compromissos com sua saúde física e mental.

M. I. S. B concebe um exemplo significativo de mudança de estilo de vida, salientando também o valor da interdisciplinaridade no caso:

“Desde moça que eu fumava. Eu sabia que o cigarro não era bom pra saúde, mas era difícil parar! O médico me falava que eu devia parar. Eu até parava, mas depois voltava. Quando iniciei o Pilates ficava até sem fôlego (risos). Mas saía daqui e 
já acendia um cigarro, escondido do professor e das minhas colegas. Se fosse só o Pilates acho que eu continuaria fumando. Mas depois nas aulas a gente foi conversando sobre a saúde e eu me convenci de parar. Não foi fácil. Mas parei e tô feliz assim".

Segundo Buss (2000), o trabalho interdisciplinar facilita transformações no campo biopsicossocial, sobretudo em sentido afetivo. Com ela há o ganho da abordagem holística em saúde, o que permite tratar o caso de forma ampla e dinâmica.

\section{Considerações finais}

Por meio da oralidade foi possível "desocultar" peculiaridades concernentes aos benefícios do Programa de Atividade Física conexo a um Programa de Atenção Psicológica, fundamentado nas Modalidades da Prática Psicológica sobre a qualidade de vida de dez idosas. Sendo assim, considerando que a prática do Pilates intensifica a consciência da movimentação corporal, contrologia, a nível respiratório, muscular e articular sua combinação com as atividades psicossociais possibilitou abranger a saúde das idosas em sentido integral. Isso significa dizer que desenvolvidas por este estudo instituíram um ambiente hábil para que alternativas à resolução de necessidades pessoais em caráter físico, funcional, emocional e social fossem trabalhadas sob a forma cooperativa e lúdica. Prontamente, as medidas conduziram o grupo à percepção e à consciência da diferença condicional entre "ser" idoso e "estar" idoso.

Uma importante conquista da ação interdisciplinar incidiu no incentivo ao exercício reflexivo junto às idosas de que a qualidade de vida e o bem-estar, ao longo do processo do envelhecimento, não dependem exclusivamente das políticas públicas de saúde ou do consumo de medicamentos. Mas que elas também se encontram nas mãos de cada cidadão. Em sentido metodológico, o estudo demonstrou a importância e dimensão da inclusão do Psicólogo junto às atividades do professor de Educação Física. Sendo assim, esta ação contribuiu para o desenvolvimento de novas formas de pensar o Ensino, a Pesquisa e a Extensão na Universidade Federal do Vale do São Francisco.

Considera-se como possível limitação deste estudo, na época de sua realização, ano de 2013, a presença de uma só aluna de Psicologia para o desenvolvimento das dinâmicas psicossociais com 50 idosas do PAF. Entretanto, pode-se afirmar que atualmente a equipe "Pilates e - Idoso" dispõem de 15 acadêmicos e dois professores do curso de Psicologia da Univasf. Isso demonstra avanços significativos na relação entre áreas do conhecimento, bem como na qualificação do 
cuidado e da atenção com os cidadãos idosos da cidade de PetrolinaPE.

\section{Referências}

Alves, R. V., Mota, J., Costa, M. D. C., \& Alves, J. G. B. (2004). Aptidão física relacionada à saúde de idosos: influência da hidroginástica. Revista Brasileira de Medicina do Esporte, 10(1), 31-37.

Araújo, M. O. P. H., \& Ceolim, M. F. (2007). Avaliação do grau de independência de idosos residentes em instituições de longa permanência. Revista da Escola de Enfermagem da USP, 41(3), 378-385.

Araújo, L. F., Coutinho, M. P. L., \& Carvalho, V. A. M. L. (2005). Representações sociais da velhice entre idosos que participam de grupos de convivência. Psicologia Ciência e Profissão, 25(1), 118-131.

Bardin, L. (2004). Análise de Conteúdo. 3 ed. Lisboa: Edições 70.

Barreto, C. L. B. T. (2009). Modalidades de Prática Psicológica Clínica: Atenção psicológica e atitude Fenomenológica Hermenêutica. IX Simpósio Nacional de Práticas Psicológicas em Instituições Atenção Psicológica: Fundamentos, Pesquisa e Prática, Recife: Unicap. Recuperado em maio 17, 2015, de http: //laclife. wordpress.com/textos-e-anais-eletronicos

Bastos, A. B. B. I. (2010). A técnica de grupos-operativos à luz de Pichon-Riviére e Henri Wallon. Psicólogo informação, 14(14), 160-169.

Batista, P. S. S. (2010). A valorização da espiritualidade nas práticas de educação popular em saúde desenvolvidas na atenção básica. Revista Eletrônica de Comunicação, Informação \& I novação em Saúde, 4(3), 49-55.

Benedetti, T. R., Gonçalves, L. H. T., \& Mota, J. P. S. (2007). Uma proposta de política pública de atividade física para idosos. Texto Contexto Enfermagem, 16(3), 387-398.

Benedetti, T. R., Mazo, G. Z., \& Borges, L. J. (2012). Condições de saúde e nível de atividade física em idosos participantes e não participantes de grupos de convivência de Florianópolis. Revista Ciência \& Saúde Coletiva, 17(8): 2087-2093.

Benevides, D. S., Pinto, A. G. A., \& Cavalcante, C.M. (2010). Cuidado em saúde mental por meio de grupos terapêuticos de um hospital-dia: perspectivas dos trabalhadores de saúde. Interface Comunidade Saúde Educação, Botucatu, 14(32), 127138.

Braga Cuba, C. M. G., Carvalho, A. S., Evangelista, K. B., \& Camara, A. M. (2015, agosto). A assessoria em uma vivência de amizade 
intergeracional com Idosos da UNATI/UERJ. Anais da VII Jornada de Políticas Públicas. São Luis, MA, Brasil. Recuperado em 18 janeiro, 2016, de http://www.joinpp.ufma.br/jornadas/joinpp2015/pdfs/eixo6/aassessoria-em-uma-vivencia-de-amizade-intergeracional-comidosos-da-unati-uerj.pdf

Borges, C. C., \& Magalhães, A. S. (2007). Laços intergeracionais no contexto contemporâneo. Estudos de Psicologia, 16(2), 171177.

Buss, P. M. (2000). Promoção da saúde e qualidade de vida. Ciência \& Saúde Coletiva, 24(1), 163-177.

Cancela, D. M. G. (2007). O processo do Envelhecimento. Porto: Universidade Lusíada do Porto. Recuperado em 15 janeiro, 2016, de http://www.psicologia.pt/artigos/textos/TL0097.pdf

Chaim, J., Raimundo, M. E., Sant'anna, F. C. A., \& Yuaso, D. R. (2010). Prática regular de atividade física e sedentarismo: influência na qualidade de vida de idosas. Revista Brasileira de Ciências do Envelhecimento Humano, 7(2),198-209.

Chaimowicz, F. (1997). A saúde dos idosos brasileiros às vésperas do século XXI: problemas, projeções e alternativas. Revista Saúde Pública, 31(2), 184-200.

Coriolano Appell, I. P., Romo Pérez, V., Nascimento, M. M., \& Appell Coriolano, H. J. (2012). The Pilates method to improve body balance in the elderly. Archives of Exercise in Health and Disease, 3(3), 188-93.

Cupertino, C. (2006). Criação e formação: A oficina de criatividade revisitada. VI Simpósio Nacional de Práticas Psicológicas em Instituições. Psicologia e Políticas Públicas. Recuperado em 20 maio, 2015, de http://www.christinacupertino.com.br/publicacoes_exibe.asp?id $=44$

Flick, U. (2009a). Desenho da Pesquisa Qualitativa. 3 ed. Porto Alegre: Artmed.

Flick, U. (2009b). Introdução à Pesquisa Qualitativa. 3 ed. Porto Alegre: Artmed.

Gattás, M. L. B. \& Furegato, A. R. F. (2006). Interdisciplinaridade: uma Contextualização. Acta Paulista de Enfermagem, 19(3), 323-327.

Gibbs, G. (2009). Análise de Dados Qualitativos. Porto Alegre: Artmed.

Lamnek, S. (1989). Qualitative Sozialforschung, Band 2: Methoden und Techniken. München: Psychologie Verlags Union.

Latey, P. (2001). The Pilates method: history and philosophy. Journal of Bodywork and Movement Therapies, 5(4), 275-282. 
Leloup, J. Y., \& Hennezel, M. (2003). A arte de morrer: tradições religiosas e espiritualidade humanista diante da morte na atualidade. 6 ed. Petrópolis: Vozes.

Lima, L. C. V., \& Bittar, C. M. L. (2012). A percepção de qualidade de vida em idoso: um estudo exploratório. Revista Brasileira de Qualidade de Vida, 4(2), 1-11.

Lima, P. M. R., \& Coelho, V. L. D. (2011). Arte de envelhecer: um estudo exploratório sobre a história de vida e o envelhecimento. Psicologia Ciência e Profissão, 31(1), 4-19.

Luz, M. M. C., \& Amatuzzi, M. M. (2008). Vivências de felicidade de pessoas idosas. Estudos de Psicologia, 25(2), 303-307.

Matsudo, S. M. (2006). Atividade física na promoção da saúde e qualidade de vida no envelhecimento. Revista Brasileira de Educação Física e Esporte, 20(5), 135-137.

Muscolino, J. E., \& Cipriani, S. (2004). Pilates and the "powerhouse", Jornal Bodywork and Movement Therapies, 8(1), 15-24.

Nascimento, M. M., Carvalho, E. C. F., Souza, J. M. R. S., \& Andrade, N. L. (2015) Programa interdisciplinar "PAF \& PAPP": Concepções metodológicas para a atenção e o cuidado ao idoso e a formação acadêmica. Estudos Interdisciplinares sobre 0 Envelhecimento Humano, 20(1), 325-337.

Nery, A. L. (1993). Qualidade de Vida e Idade Madura. Campinas: Papirus Editora.

Neri, A. L. (2004). Contribuições da psicologia ao estudo e à intervenção no campo da velhice. Revista Brasileira de Ciências do Envelhecimento Humano, 1(1), 69-80.

Pelizzoli, M. L. (2014). Visão Histórica e Sistêmica: Bases para o paradigma integrativo em saúde. In Alexandre F. Barreto (Orgs.). Práticas Integrativas em Saúde: Proposições Teóricas e Experiências na Saúde e Educação. (pp. 23-48). Recife: Editora UFPE.

Pereira, J. R. P., \& Okuma, S. (2009). Perfil dos ingressantes de um programa de educação física para idosos e os motivos da adesão inicial. Revista Brasileira de Educação Física e Esporte, 23(4), 319-334.

Pilates, J. H. (1998). Your health. A corrective system of exercising revolutionizes the entire field of physical education. Encline, NV: Presentation Dynamics.

Rauchbach, R. (2001). A Atividade Física para 3o Idade: Envelhecimento ativo, uma proposta para a vida. Londrina: Midiograf.

Rizzolli, D., \& Surdi, A. C. (2010). Percepção dos idosos sobre grupos de terceira idade. Revista Brasileira de Geriatria e Gerontologia, 13(2), 225-233.

Rowe, J. W., \& Kahn, R. L. (1997). Successful aging. Gerontological, $37(4), 433-40$. 
Salin, M. S., Mazo, G. Z., Cardoso, A. S., \& Garcia, G. S. (2011). Atividade Física para idosos: diretrizes para implantação de programas e ações. Revista Brasileira de Geriatria e Gerontologia, 14(2), 197-208.

Santos, P. M., Souza, B. Z., Marinho, A., \& Mazo, G. Z. (2013). Percepção de qualidade de vida entre idosos jovens e longevos praticantes de hidroginástica. Revista Brasileira de Qualidade de Vida, 5(1), 1-11.

Seidl, E. M. F., \& Zannon, C. M. L. C. (2004). Qualidade de vida e saúde: aspectos conceituais e metodológicos. Cadernos de Saúde Pública, 20(2): 580-588.

Serbim, A. K., \& Figueiredo, A. E. P. L. (2011). Qualidade de vida de idosos em um grupo de convivência. Scientia Medica, 21(4): 166-172.

Spirduso, W. W. (2005). Dimensões físicas do envelhecimento. Editora Manole Ltda.

Stake, R. E. (2011). Pesquisa Qualitativa: estudando como as coisas funcionam. Porto Alegre: Penso.

Vasconcelos, E. M. (2004). A espiritualidade na educação popular em saúde. Revista APS, 7(2): 110-118.

Veras, R. (2009). Envelhecimento populacional contemporâneo: demandas, desafios e inovações. Revista de Saúde Pública, 43(3), 548-554.

Vieira, C. R. L., \& Boeckel, M. G. (2012). (Qual) idade de vida: Intervenção Psicológica junto a grupo da terceira idade. Revista de Estudo Interdisciplinar do Envelhecimento, 17(1), 138-199.

The WHOQOL Group (1995). The World Health Organization quality of life assessment (WHOQOL): position paper from the World Health Organization. Social Science and Medicine, 41(10), 1403-1409.

Yokoyama, C. E., Carvalho, R. S., \& Vizzotto, M. M. (2006). Qualidade de vida na velhice segundo a percepção de idosos frequentadores de um centro de referência. Psicólogo in Formação, 10(10), 61-62.

\section{Endereço para correspondência}

Emília Cristina Ferreira de Carvalho

Universidade Federal do Vale do São Francisco - UNIVASF-PE

Colegiado de Psicologia da Universidade Federal do Vale do São Francisco - UNIVASF

Endereço eletrônico: emiliacavalho02@hotmail.com

\section{Marcelo de Maio Nascimento}

Universidade Federal do Vale do São Francisco - UNIVASF-PE

Colegiado de Educação Física da Universidade Federal do Vale do São Francisco UNIVASF

Endereço eletrônico: marcelo.nascimento@univasf.edu.br

Recebido em: 18/11/2014

Reformulado em: 27/01/2016 
Aceito para publicação em: 10/03/2016

\section{Notas}

* Psicóloga formada pela Universidade Federal do Vale do São Francisco-UNIVASF, Petrolina, Brasil.

** Professor Adjunto do curso de Educação Física da Universidade Federal do Vale do São Francisco-UNIVASF, Petrolina, Brasil. Doutor em Ciências do Movimento Humano pela Universidade de Esportes de Colónia, Alemanha. 